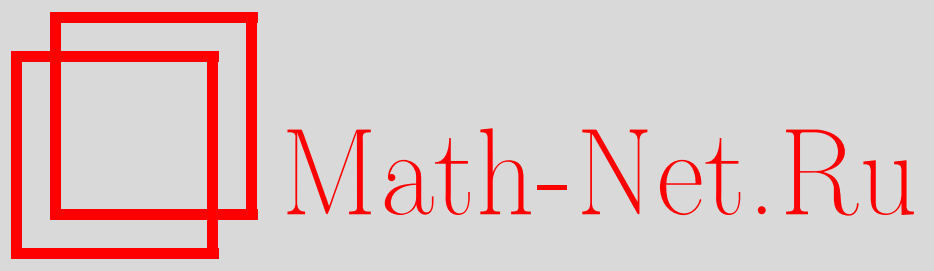

М. Бойти, Ф. Пемпинелли, А. К. Погребков, Б. Принари, Построение расширенной резольвенты оператора теплопроводности с помощью сплетающих преобразований, ТМФ, 2009, том 159, номер 3, 364-378

DOI: https://doi.org/10.4213/tmf6356

Использование Общероссийского математического портала Math-Net.Ru подразумевает, что вы прочитали и согласны с пользовательским соглашением http: //www . mathnet.ru/rus/agreement

Параметры загрузки:

IP : 52.87 .193 .239

26 апреля 2023 г., 03:44:53

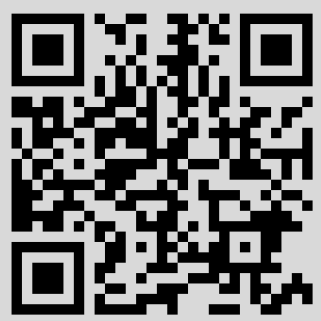




\title{
ПОСТРОЕНИЕ РАСШИРЕННОЙ РЕЗОЛЬВЕНТЫ ОПЕРАТОРА ТЕПЛОПРОВОДНОСТИ С ПОМОЩЬЮ СПЛЕТАЮЩИХ ПРЕОБРАЗОВАНИЙ
}

\begin{abstract}
Введены сплетающие преобразования для оператора теплопроводности. Они позволяют одновременно наложить по Дарбу $N$ солитонов на общий гладкий убывающий на бесконечности потенциал и построить соответствующие решения Йоста. Эти сплетающие операторы также использованы для исследования проблемы существования расширенной резольвенты. Детально рассмотрены существование и единственность расширенной резольвенты в случае $N$ солитонов с $N$ "входящими" лучами и одним "исходящим" лучом.
\end{abstract}

Ключевые слова: преобразование Дарбу, многомерный солитон, аннигилятор.

\section{1. ВВЕДЕНИЕ}

Уравнение Кадомцева-Петвиашвили в версии, называемой КПII,

$$
\left(u_{t}-6 u u_{x_{1}}+u_{x_{1} x_{1} x_{1}}\right)_{x_{1}}=-3 u_{x_{2} x_{2}}
$$

является $(2+1)$-мерным обобщением знаменитого уравнения Кортевега-де Фриза $($ КдФ). Как следствие уравнение КПІІ допускает решения, ведущие себя на пространственной бесконечности подобно решениям уравнения КдФ. Например, если $u_{1}\left(t, x_{1}\right)$ удовлетворяет уравнению Кд $\Phi$, то $u\left(t, x_{1}, x_{2}\right)=u_{1}\left(t, x_{1}+\mu x_{2}-3 \mu^{2} t\right)$ является решением уравнения КПІІ для произвольной константы $\mu \in \mathbb{R}$. Таким образом, важно рассматривать решения уравнения (1.1), которые убывают на бесконечности во всех направлениях за исключением конечного числа одномерных лучей с поведением типа $u_{1}$. Даже несмотря на то что уравнение KПII известно как интегрируемое уравнение более трех десятков лет [1], [2], его общая теория (включающая такие неубывающие решения) далека от завершения. Так, задача Коши для уравнения KПII с быстроубывающими начальными данными была решена в работах [3], [4]

*Dipartmento di Fisica, Universitá del Salento and INFN, I-73100 Lecce, Italy.

${ }^{\dagger}$ Математический институт им. В. А. Стеклова РАН, Москва, Россия. E-mail: pogreb@mi.ras.ru 
с помощью метода обратной задачи рассеяния (МОЗР), основанного на спектральном анализе оператора теплопроводности

$$
\mathcal{L}\left(x, \partial_{x}\right)=-\partial_{x_{2}}+\partial_{x_{1}}^{2}-u(x), \quad x=\left(x_{1}, x_{2}\right),
$$

задающего вспомогательную линейную задачу для уравнения КПІІ. Стандартный подход к спектральной теории оператора (1.2) основан на интегральных уравнениях для решения Йоста $\Phi(x, \mathbf{k})$ или для $\Psi(x, \mathbf{k})$ - решения Йоста дуального оператора $\mathcal{L}^{\mathrm{d}}$, где $\mathbf{k} \in \mathbb{C}-$ спектральный параметр. Однако известно, что в случае потенциалов с одномерным асимптотическим поведением данные интегральные уравнения плохо определены.

Для преодоления этих трудностей в работах [5]-[14] был предложен резольвентный подход. В рамках этого подхода этом подходе вводится пространство операторов $A(q)$ с ядрами $A\left(x, x^{\prime} ; q\right)$, принадлежащими пространству обобщенных функций умеренного роста от переменных $x, x^{\prime}, q \in \mathbb{R}^{2}$. Обычные дифференциальные операторы $\mathcal{L}\left(x, \partial_{x}\right)$ погружаются в это пространство как операторы с ядрами

$$
L\left(x, x^{\prime} ; q\right) \equiv \mathcal{L}\left(x, \partial_{x}+q\right) \delta\left(x-x^{\prime}\right)
$$

В силу дополнительной зависимости от параметра $q$ эти операторы называются расширенными. В этом пространстве было введено обобщение резольвенты дифференциального оператора, называемое расширенной резольвентой, которое позволило рассматривать спектральную теорию операторов с нетривиальным асимптотическим поведением на пространственной бесконечности.

В работах [11]-[14] мы рассмотрели нестационарный оператор Шредингера и оператор теплопроводности с потенциалами, не убывающими только по одному направлению. Первым шагом в решении задачи было погружение чисто одномерной спектральной теории в двумерную посредством построения двумерной расширенной резольвенты для оператора с потенциалом $u(x) \equiv u_{1}\left(x_{1}\right)$. Второй шаг состоял в одевании этой резольвенты с помощью произвольного двумерного возмущения потенциала $u_{1}$. В конечном итоге все математические объекты, относящиеся к МОЗР, были выведены из расширенной резольвенты путем редукции, что позволило сформулировать прямую и обратную задачи. Отметим также, что стандартная спектральная теория оператора теплопроводности для случая потенциала, не убывающего в одном пространственном направлении, была развита в работе [15], однако при некоторых специальных и неявных условиях на потенциал.

В настоящей работе мы рассматриваем заметно более сложную проблему: случай потенциала $u$, не убывающего по нескольким непаралельным лучам. В этом случае нет аналогии с одномерной задачей, и вся теория должна быть построена непосредственно, без погружения одномерных величин в два измерения. Следовательно, мы должны рассматривать действительно двумерные потенциалы напрямую, как это уже было сделано в работах [16], [17] для нестационарного оператора Шредингера. Фактически мы используем процедуру, которая была успешно применена и в том случае. А именно, для построения потенциала, отвечающего $N$ солитонам, наложенным на общий гладкий убывающий потенциал, а также для построения соответствующих решений Йоста, мы вместо рекурсивного применения 
(бинарных) преобразований Дарбу строим эти величины напрямую, используя сплетающие преобразования. Таким способом мы приходим к потенциалам и решениям Йоста, полученным ранее в статье [11] с помощью рекурсивной процедуры, а также к альтернативным представлениям чисто солитонных потенциалов, полученным в работах [18]-[22] с применением $\tau$-функции. Здесь уместно отметить, что оператор теплопроводности не является самодуальным, а потому сингулярное поведение левого и правого сплетающих операторов не обязано быть согласованным. Это объясняет, почему структура $N$-солитонного решения для уравнения КПІІ намного более богата, чем для уравнения KПІ. В частности, $N$-солитонное решение может иметь различное число "входящих" и "исходящих" (в смысле $x_{2} \rightarrow-\infty$ или $x_{2} \rightarrow+\infty$ ) лучей.

Статья организована следующим образом. В разделе 2 мы кратко излагаем некоторые аспекты и основные идеи метода расширенной резольвенты, основываясь на примере оператора (1.2) с гладким, быстроубывающим потенциалом $u(x)$. За дальнейшими деталями мы отсылаем заинтересованного читателя к работам [5]-[9]. В разделе 3 мы вводим сплетающие операторы, т.е. операторы, которые "сплетают" оператор $L$ - расширение в смысле $(1.3)$ оператора $\mathcal{L}$ из $(1.2)$ - и оператор $L^{\prime}$ того же типа с потенциалом $u^{\prime}$, описывающим $N$ солитонов, наложенных по Дарбу на фоновый потенциал $u$. В разделе 4 , применяя эти сплетающие операторы, мы выводим при условии существования резольвенты $M^{\prime}(q)$ явное выражение для ее ядра $M^{\prime}\left(x, x^{\prime} ; q\right)$. Таким образом, проблема существования резольвенты $M^{\prime}(q)$ сводится к проблеме нахождения области на $q$-плоскости, где ядро является распределением умеренного роста. Эта задача оказалась более тяжелой, чем в случае нестационарного оператора Шредингера, а потому в разделе 5 мы сначала рассматриваем чисто $N$-солитонные потенциалы, а затем в разделе 6 - специальный простой подкласс $N$-солитонных потенциалов с $N$ “входящими" лучами и только одним "исходящим" лучом. Мы доказываем, что для $N>1$ резольвента $M^{\prime}(q)$ существует только в области $q$-плоскости, внешней к некоторому многоугольнику с $N+1$ сторонами. Более конкретно, мы доказываем, что существует такое значение $\mathbf{k}_{0}$ спектрального параметра $\mathbf{k}$, для которого дуальное решение Йоста $\Psi^{\prime}(x, \mathbf{k})$ удовлетворяет условию, что $\Psi^{\prime}\left(x, \mathbf{k}_{0}\right) e^{q_{1} x_{1}+q_{2} x_{2}}$ убывает на $x$-плоскости экспоненциально для любого значения $q$, принадлежащего этому многоугольнику. Отсюда мы заключаем, что внутри этого многоугольника твистованный оператор $L^{\prime}(q)$ обладает левым аннигилятором и не может иметь правого обратного. Однако функция Грина оператора $\mathcal{L}^{\prime}$ существует и может быть однозначно получена с помощью редукции $M^{\prime}(q)$. В последующей публикации мы планируем рассмотреть общий случай произвольного числа входящих и исходящих лучей и прояснить роль этих аннигиляторов в спектральной теории таких потенциалов.

\section{2. ФОНОВАЯ ТЕОРИЯ}

Введем пространство расширенных операторов $A(q)$, т.е. операторов с ядром $A\left(x, x^{\prime} ; q\right)$, принадлежащим пространству $\mathcal{S}^{\prime}$ обобщенных функций умеренного роста от шести вещественных переменных $x=\left(x_{1}, x_{2}\right), x^{\prime}=\left(x_{1}^{\prime}, x_{2}^{\prime}\right)$ и $q=\left(q_{1}, q_{2}\right)$. Для двух расширенных операторов $A(q)$ и $B(q)$ с ядрами $A\left(x, x^{\prime} ; q\right)$ и $B\left(x, x^{\prime} ; q\right)$ мы вводим 
закон композиции

$$
(A B)\left(x, x^{\prime} ; q\right)=\int d x^{\prime \prime} A\left(x, x^{\prime \prime} ; q\right) B\left(x^{\prime \prime}, x^{\prime} ; q\right)
$$

предполагая, что интеграл существует в смысле распределений. Оператор $A$ может иметь обратный $A^{-1}$ в смысле этой композиции: $A A^{-1}=I$ и $A^{-1} A=I$, где $I-$ единичный оператор, т.е. оператор с ядром $I\left(x, x^{\prime} ; q\right)=\delta\left(x_{1}-x_{1}^{\prime}\right) \delta\left(x_{2}-x_{2}^{\prime}\right)$. Специальный подкласс расширенных операторов дается расширениями $L(q)$ дифференциальных операторов, определенными в $(1.3)$, где $\mathcal{L}\left(x, \partial_{x}\right)$ означает дифференциальный оператор, коэффициенты которого суть гладкие функции от $x$. Поставим в соответствие каждому оператору $A(q)$ с ядром $A\left(x, x^{\prime} ; q\right)$ его "хет"-ядро

$$
\hat{A}\left(x, x^{\prime} ; q\right)=e^{q\left(x-x^{\prime}\right)} A\left(x, x^{\prime} ; q\right),
$$

где $q x=q_{1} x_{1}+q_{2} x_{2}$. Для дифференциального оператора $L(q)$ эта процедура является обратной к расширению, введенному в $(1.3)$, т.е. $\hat{L}\left(x, x^{\prime} ; q\right)=L\left(x, x^{\prime}\right)$. Заметим, однако, что, поскольку на ядра операторов $A$ налагается лишь условие принадлежности пространству распределений, в общем случае хет-ядро $\hat{A}$ зависит от $q$ и не обязано быть ограниченным. Укажем также, что оператор $A^{*}(q)$, сопряженный оператору $A(q)$, задается ядром

$$
A^{*}\left(x, x^{\prime} ; q\right)=\overline{A\left(x, x^{\prime} ; q\right)}
$$

так что самосопряженный оператор - это оператор с вещественным ядром.

Удобно ввести задаваемое преобразованием Фурье представление оператора $A(q)$ в $p$-пространстве:

$$
A(p ; \mathbf{q})=\frac{1}{(2 \pi)^{2}} \int d x \int d x^{\prime} e^{i\left(p+\mathbf{q}_{\Re}\right) x-i \mathbf{q}_{\Re} x^{\prime}} A\left(x, x^{\prime} ; \mathbf{q}_{\Im}\right),
$$

где $p=\left(p_{1}, p_{2}\right) \in \mathbb{R}^{2}$ и мы ввели двумерный комплексный вектор

$$
\mathbf{q}=\mathbf{q}_{\Re}+i \mathbf{q}_{\Im}, \quad q \equiv \mathbf{q}_{\Im}, \quad \mathbf{q}_{\Re}, \mathbf{q}_{\Im} \in \mathbb{R}^{2} .
$$

Композиция (2.1) в p-пространстве принимает вид “сдвинутой” свертки

$$
(A B)(p ; \mathbf{q})=\int d p^{\prime} A\left(p-p^{\prime} ; \mathbf{q}+p^{\prime}\right) B\left(p^{\prime} ; \mathbf{q}\right) .
$$

С учетом сделанных определений расширение оператора теплопроводности (1.2) имеет вид

$$
L=L_{0}-u, \quad u\left(x, x^{\prime} ; q\right)=u(x) \delta\left(x-x^{\prime}\right),
$$

где $L_{0}$ является расширением в смысле $(1.3)$ дифференциальной части $\mathcal{L}_{0}$ оператора теплопроводности (1.2) и в силу (2.4) имеет в $p$-пространстве ядро, задаваемое равенством

$$
L_{0}(p ; \mathbf{q})=\left(i \mathbf{q}_{2}-\mathbf{q}_{1}^{2}\right) \delta(p)
$$


Основной объект нашего исследования - расширенная резольвента (или просто резольвента, для краткости) $M(q)$ оператора $L(q)$, т.е. оператор, обратный к $L$ в смысле композиции (2.1) (или (2.6)):

$$
L M=M L=I .
$$

Он может быть определен как решение интегральных уравнений

$$
M=M_{0}+M_{0} u M, \quad M=M_{0}+u M M_{0},
$$

где $M_{0}$ - резольвента оператора $L_{0}$ с нулевым потенциалом (голого оператора). В силу (2.8) мы имеем для ядра этого оператора в $p$-пространстве соотношение $M_{0}(p ; \mathbf{q})=\delta(p) /\left(i \mathbf{q}_{2}-\mathbf{q}_{1}^{2}\right)$. В случае быстроубывающего потенциала $u(x)$ существование и единственность решения уравнений (2.10) можно доказать по аналогии с работой [4]. Заметим, что для вещественного потенциала $u(x)$ как $L$, так и $M$ самосопряжены в смысле определения (2.3).

В силу (2.6) и данной выше явной формы $M_{0}(p ; \mathbf{q})$ интегральные уравнения $(2.10)$, записанные в $p$-пространстве, показывают, что ядро $M(p ; \mathbf{q})$ сингулярно при $\mathbf{q}_{2}=$ $-i \mathbf{q}_{1}^{2}$ и при $\mathbf{q}_{2}+p_{2}=-i\left(\mathbf{q}_{1}+p_{1}\right)^{2}$. Поэтому естественно ввести следующие транкированые и редуцированные значения резольвенты:

$$
\begin{aligned}
\nu(p ; \mathbf{q}) & =\left.\left(M L_{0}\right)(p ; \mathbf{q})\right|_{\mathbf{q}_{2}=-i \mathbf{q}_{1}^{2}}, \\
\omega(p ; \mathbf{q}) & =\left.\left(L_{0} M\right)(p ; \mathbf{q})\right|_{\mathbf{q}_{2}=-i\left(\mathbf{q}_{1}+p_{1}\right)^{2}-p_{2}} .
\end{aligned}
$$

Можно показать, что оператор $L(q)$ и его резольвента $M(q)$ допускают следующие билинейные представления через $\nu$ и $\omega$ :

$$
L=\nu L_{0} \omega, \quad M=\nu M_{0} \omega .
$$

Мы называем операторы $\nu$ и $\omega$ одевающими, поскольку они "одевают" голые операторы $L_{0}$ и $M_{0}$. Отметим, что $\nu(p ; \mathbf{q})$ и $\omega(p ; \mathbf{q})$ имеют асимптотическое поведение

$$
\lim _{\mathbf{q}_{1} \rightarrow \infty} \nu(p ; \mathbf{q})=\delta(p), \quad \lim _{\mathbf{q}_{1} \rightarrow \infty} \omega(p ; \mathbf{q})=\delta(p)
$$

и не зависят от $\mathbf{q}_{2}$, что при необходимости мы поясняем записью $\nu\left(p ; \mathbf{q}_{1}\right)$ и $\omega\left(p ; \mathbf{q}_{1}\right)$.

Одевающие операторы $\nu$ и $\omega$ удовлетворяют уравнениям

$$
L \nu=\nu L_{0}, \quad \omega L=L_{0} \omega
$$

и являются взаимно обратными:

$$
\omega \nu=I, \quad \nu \omega=I .
$$

Для того чтобы задать с помощью одевающих операторов решения Йоста, мы вводим следующие преобразования Фурье:

$$
\chi\left(x, \mathbf{q}_{1}\right)=\int d p e^{-i p x} \nu\left(p ; \mathbf{q}_{1}\right), \quad \xi\left(x, \mathbf{q}_{1}\right)=\int d p e^{-i p x} \omega\left(p ; \mathbf{q}_{1}-p_{1}\right) .
$$


Тогда решение Йоста и дуальное решение Йоста можно определить, соответственно, как

$$
\Phi(x, \mathbf{k})=e^{-i \mathbf{k} x_{1}-\mathbf{k}^{2} x_{2}} \chi(x, \mathbf{k}), \quad \Psi(x, \mathbf{k})=e^{i \mathbf{k} x_{1}+\mathbf{k}^{2} x_{2}} \xi(x, \mathbf{k}),
$$

где мы обозначили $\mathbf{q}_{1}$ как $\mathbf{k}$, что отвечает стандартному обозначению спектрального параметра. В силу (2.13) эти функции удовлетворяют уравнению теплопроводности и его дуальному:

$$
\left(-\partial_{x_{2}}+\partial_{x_{1}}^{2}-u(x)\right) \Phi(x, \mathbf{k})=0, \quad\left(\partial_{x_{2}}+\partial_{x_{1}}^{2}-u(x)\right) \Psi(x, \mathbf{k})=0 .
$$

\section{3. ПРЕОБРАЗОВАНИЯ ДАРБУ ПОСРЕДСТВОМ СПЛЕТАЮЩИХ ОПЕРАТОРОВ $\zeta$ И $\eta$}

Для построения двумерного потенциала, описывающего $N$ солитонов, наложенных на общий гладкий фон, мы обходим рекурсивную процедуру, использованную в работе [11] и, используя операторную формулировку, введенную в предыдущем разделе, напрямую строим окончательные величины с помощью сплетающих операторов. А именно, рассмотрим преобразование от оператора $L$ в $(2.7)$ к новому оператору того же вида

$$
L^{\prime}=L_{0}-u^{\prime}, \quad u^{\prime}\left(x, x^{\prime} ; q\right)=u^{\prime}(x) \delta\left(x-x^{\prime}\right),
$$

заданному операторной парой $\zeta, \eta$ посредством формул

$$
L^{\prime} \zeta=\zeta L, \quad \eta L^{\prime}=L \eta,
$$

“сплетающих" $L$ с $L^{\prime}$. Мы считаем потенциал $u(x)$ в $L$ вещественной, гладкой, быстроубывающей функцией и ищем самосопряженные операторы $\zeta$ и $\eta$ такие, что новый потенциал $u^{\prime}(x)$ также является вещественным и гладким. Помимо этого мы требуем, чтобы $\eta$ был левым обратным к оператору $\zeta$, т.е. удовлетворял условию

$$
\eta \zeta=I
$$

так что

$$
L=\eta L^{\prime} \zeta
$$

Для того чтобы новый потенциал $u^{\prime}(x)$ не убывал по некоторым направлениям на плоскости, два оператора $L$ и $L^{\prime}$ должны быть связаны преобразованием, более общим, чем подобие. Поэтому мы ищем сплетающие операторы, для которых произведение $\zeta \eta$ отлично от $I$. Полагая

$$
\zeta \eta=I-P,
$$

получаем, что $P$ - ортогональный самосопряженный проектор, поскольку в силу (3.3) $P^{2}=P$ и $P^{*}=P$ благодаря свойству самосопряженности $\zeta$ и $\eta$. Сплетающие операторы $\zeta$ и $\eta$ порождают новый потенциал $u^{\prime}$ посредством формул (3.2), а также новые одевающие операторы $\nu^{\prime}$ и $\omega^{\prime}$. Действительно, учтя (2.13), мы получаем на основании (3.2), что $L^{\prime} \zeta \nu=\zeta L \nu=\zeta \nu L_{0}$ и $\omega \eta L^{\prime}=\omega L \eta=L_{0} \omega \eta$. Поэтому операторы $\nu^{\prime}$ и $\omega^{\prime}$ задаются как

$$
\nu^{\prime}=\zeta \nu, \quad \omega^{\prime}=\omega \eta
$$


и удовлетворяют уравнениям

$$
L^{\prime} \nu^{\prime}=\nu^{\prime} L_{0}, \quad \omega^{\prime} L^{\prime}=L_{0} \omega^{\prime},
$$

аналогичным уравнениям (2.13) для одевающих операторов $\nu$ и $\omega$.

Отметим, что в силу (3.3) скалярное произведение этих одевающих операторов, как и исходных в (2.14), равно $I$ :

$$
\omega^{\prime} \nu^{\prime}=I
$$

Однако в отличие от $\nu$ и $\omega$ эти операторы не удовлетворяют соотношению полноты, поскольку из (2.14) и в отличие от него мы имеем в силу (3.5)

$$
\nu^{\prime} \omega^{\prime}+P=I
$$

Для получения преобразования Дарбу следует конкретизировать свойства аналитичности ядер $\zeta(p ; \mathbf{q})$ и $\eta(p ; \mathbf{q})$ сплетающих операторов по переменным q. Вследствие (2.14) и (3.6) мы имеем, что $\zeta=\nu^{\prime} \omega$ и $\eta=\nu \omega^{\prime}$, поэтому сингулярности этих ядер связаны со свойствами $\nu^{\prime}(p ; \mathbf{q})$ и $\omega^{\prime}(p ; \mathbf{q})$. Мы требуем следующее.

1. Ядра $\nu^{\prime}(p ; \mathbf{q})$ и $\omega^{\prime}(p ; \mathbf{q})$ не должны зависеть от $\mathbf{q}_{2}$ и должны иметь асимптотическое поведение $\lim _{\mathbf{q}_{1} \rightarrow \infty} \nu^{\prime}\left(p ; \mathbf{q}_{1}\right)=\delta(p)$ и $\lim _{\mathbf{q}_{1} \rightarrow \infty} \omega^{\prime}\left(p ; \mathbf{q}_{1}\right)=\delta(p)$.

2. Ядра $\nu^{\prime}\left(p ; \mathbf{q}_{1}\right)$ и $\omega^{\prime}\left(p ; \mathbf{q}_{1}\right)$ должны иметь (соответственно, правые и левые) простые полюсы по переменной $\mathbf{q}_{1}$, т.е. должны существовать нетривиальные пределы

$$
\begin{aligned}
\nu_{b_{l}}^{\prime}(p) & =\lim _{\mathbf{q}_{1} \rightarrow i b_{l}}\left(\mathbf{q}_{1}-i b_{l}\right) \nu^{\prime}\left(p ; \mathbf{q}_{1}\right), \\
\omega_{a_{j}}^{\prime}(p) & =\lim _{\mathbf{q}_{1} \rightarrow-p_{1}+i a_{j}}\left(\mathbf{q}_{1}+p_{1}-i a_{j}\right) \omega^{\prime}\left(p ; \mathbf{q}_{1}\right),
\end{aligned}
$$

где $a_{1}, \ldots, a_{N_{a}}, b_{1}, \ldots, b_{N_{b}}$ суть $N_{a}+N_{b}$ параметров, которые мы выбираем все различными и вещественными, чтобы гарантировать вещественность преобразованного потенциала.

3. Ядра $\zeta(p ; \mathbf{q})$ и $\eta(p ; \mathbf{q})$ помимо разрывов при $\mathbf{q}_{1 \Im}=b_{l}$ и $\mathbf{q}_{1 \Im}=a_{j}$, наличие которых вытекает из требования 2 , не должны иметь иных дефектов аналитичности по $\mathbf{q}_{1}$.

При этих условиях ядра операторов $\zeta, \eta$ задаются следующими равенствами

$$
\begin{aligned}
& \zeta\left(p ; \mathbf{q}_{1}\right)=\delta(p)+\sum_{l=1}^{N_{b}} \int d p^{\prime} \frac{\nu_{b_{l}}^{\prime}\left(p-p^{\prime}\right) \omega\left(p^{\prime} ; i b_{l}-p_{1}^{\prime}\right)}{\mathbf{q}_{1}+p_{1}^{\prime}-i b_{l}} \\
& \eta\left(p ; \mathbf{q}_{1}\right)=\delta(p)+\sum_{j=1}^{N_{a}} \int d p^{\prime} \frac{\nu\left(p-p^{\prime} ; i a_{j}\right) \omega_{a_{j}}^{\prime}\left(p^{\prime}\right)}{\mathbf{q}_{1}+p_{1}^{\prime}-i a_{j}}
\end{aligned}
$$

где использованы обозначения (3.10) и (3.11). Пусть теперь $\chi^{\prime}(x, \mathbf{k})$ и $\xi^{\prime}(p ; \mathbf{k})$ означают функции, определенные через одевающие операторы $\nu^{\prime}$ и $\omega^{\prime}$ по аналогии с (2.15), 
a $\chi_{b_{l}}^{\prime}(x)$ и $\xi_{a_{j}}^{\prime}(x)$ суть их вычеты (cp. (3.10) и (3.11)). Тогда легко показать, что уравнение (3.3) эквивалентно набору уравнений

$$
\begin{array}{ll}
\chi_{b_{l}}^{\prime}(x)=-i \sum_{j=1}^{N_{a}} \chi\left(x, i a_{j}\right) m_{j l}(x), & l=1, \ldots, N_{b}, \\
\xi_{a_{j}}^{\prime}(x)=i \sum_{l=1}^{N_{b}} m_{j l}(x) \xi\left(x, i b_{l}\right), & j=1, \ldots, N_{a},
\end{array}
$$

где $m(x)-\left(N_{a} \times N_{b}\right)$-матрица с элементами

$$
m_{j l}(x)=\left.\int_{x_{1}}^{\left(a_{j}-b_{l}\right) \infty} d y_{1} e^{\left(a_{j}-b_{l}\right)\left(x_{1}-y_{1}\right)} \xi_{a_{j}}^{\prime}(y) \chi_{b_{l}}^{\prime}(y)\right|_{y_{2}=x_{2}},
$$

которая хорошо определена для ограниченных $\chi_{b_{l}}^{\prime}$ и $\xi_{a_{j}}^{\prime}$ и для элементов которой имеет место уравнение

$$
\partial_{x_{1}} m_{j l}(x)=\left(a_{j}-b_{l}\right) m_{j l}(x)-\xi_{a_{j}}^{\prime}(x) \chi_{b_{l}}^{\prime}(x) .
$$

В дополнение из (3.1) и (3.2) мы получаем для преобразованного потенциала $u^{\prime}(x)$ следующее равенство:

$$
u^{\prime}(x)=u(x)-2 \partial_{x_{1}} \sum_{j=1}^{N_{a}} \sum_{l=1}^{N_{b}} \xi\left(x, i b_{l}\right) \chi\left(x, i a_{j}\right) m_{j l}(x) .
$$

Тогда в силу (3.12) и (3.13) для определения $\zeta$ и $\eta$ следует конкретизировать $\nu_{b_{l}}^{\prime}$ и $\omega_{a_{j}}^{\prime}$, т.е. $\chi_{b_{l}}^{\prime}(x)$ и $\xi_{a_{j}}^{\prime}(x)$. Вследствие (3.14) и (3.15) это означает, что нам следует определить матрицу $m(x)$. Подставляя равенства (3.14) и (3.15) в (3.17) и используя (2.16), мы выписываем следующее уравнение для матрицы $\widehat{m}(x)$ с элементами $\widehat{m}_{j l}(x)=e^{\left(b_{l}-a_{j}\right)\left(x_{1}+\left(b_{l}+a_{j}\right) x_{2}\right)} m_{j l}(x)$ :

$$
\partial_{x_{1}} \widehat{m}_{j l}(x)=-\sum_{j^{\prime}=1}^{N_{a}} \sum_{l^{\prime}=1}^{N_{b}} \widehat{m}_{j l^{\prime}}(x) \Psi\left(x, i b_{l^{\prime}}\right) \Phi\left(x, i a_{j^{\prime}}\right) \widehat{m}_{j^{\prime} l}(x) .
$$

Опуская детали, сразу приведем здесь вид $\widehat{m}(x)$ в двух эквивалентных формах

$$
\widehat{m}(x)=\left(E_{N_{a}}+c \mathcal{F}(x)\right)^{-1} c=c\left(E_{N_{b}}+\mathcal{F}(x) c\right)^{-1},
$$

где $c$ - произвольная вещественная постоянная $\left(N_{a} \times N_{b}\right)$-матрица, $E_{N_{a}}$ и $E_{N_{b}}$ суть единичные $\left(N_{a} \times N_{a}\right)$ - и $\left(N_{b} \times N_{b}\right)$-матрицы соответственно, а $\mathcal{F}(x)-\left(N_{b} \times N_{a}\right)$-матрица с элементами $\mathcal{F}_{l j}(x)=\mathcal{F}\left(x, i b_{l}, i a_{j}\right)$, где

$$
\mathcal{F}\left(x, \mathbf{k}, \mathbf{k}^{\prime}\right)=\left.\int_{\left(\mathbf{k}_{\Im}-\mathbf{k}_{\Im}^{\prime}\right) \infty}^{x_{1}} d x_{1}^{\prime} \Psi\left(x^{\prime}, \mathbf{k}\right) \Phi\left(x^{\prime}, \mathbf{k}^{\prime}\right)\right|_{x_{2}^{\prime}=x_{2}} .
$$

Теперь потенциал (3.18) также может быть записан в двух эквивалентных форmax:

$$
u^{\prime}(x)=u(x)-2 \partial_{x_{1}}^{2} \ln \operatorname{det}\left(E_{N_{b}}+\mathcal{F} c\right)=u(x)-2 \partial_{x_{1}}^{2} \ln \operatorname{det}\left(E_{N_{a}}+c \mathcal{F}\right) .
$$


Подставляя найденную выше матрицу $m$ в (3.14), (3.15), а затем полученные равенства в (3.12), (3.13), мы выводим явную форму для одевающих операторов $\zeta$ и $\eta$. Наконец, из (3.6), используя равенство (2.16) и его аналог для преобразованных (штрихованных) решения Йоста и дуального решения Йоста, мы получаем для них по два эквивалентных представления:

$$
\begin{aligned}
\Phi^{\prime}(x, \mathbf{k}) & =\Phi(x, \mathbf{k})-\Phi(x, i a)\left(E_{N_{a}}+c \mathcal{F}(x)\right)^{-1} c \mathcal{F}(x, i b, \mathbf{k})= \\
& =\Phi(x, \mathbf{k})-\Phi(x, i a) c\left(E_{N_{b}}+\mathcal{F}(x) c\right)^{-1} \mathcal{F}(x, i b, \mathbf{k}), \\
\Psi^{\prime}(x, \mathbf{k}) & =\Psi(x, \mathbf{k})-\mathcal{F}(x, \mathbf{k}, i a)\left(E_{N_{a}}+c \mathcal{F}(x)\right)^{-1} c \Psi(x, i b)= \\
& =\Psi(x, \mathbf{k})-\mathcal{F}(x, \mathbf{k}, i a) c\left(E_{N_{b}}+\mathcal{F}(x) c\right)^{-1} \Psi(x, i b),
\end{aligned}
$$

где $j=1, \ldots, N_{a}, l=1, \ldots, N_{b}$,

$$
\begin{aligned}
\Phi(x, i a) & =\operatorname{diag}\left\{\Phi\left(x, i a_{j}\right)\right\}, & \Psi(x, i b) & =\operatorname{diag}\left\{\Psi\left(x, i b_{l}\right)\right\}, \\
\mathcal{F}(x, \mathbf{k}, i a) & =\operatorname{diag}\left\{\mathcal{F}\left(x, \mathbf{k}, i a_{j}\right)\right\}, & \mathcal{F}(x, i b, \mathbf{k}) & =\operatorname{diag}\left\{\mathcal{F}\left(x, i b_{l}, \mathbf{k}\right)\right\}
\end{aligned}
$$

и где может быть использовано любое из двух представлений в (3.23) и (3.24). Легко видеть, что как $\Phi^{\prime}(x, \mathbf{k})$, так и $\Psi^{\prime}(x, \mathbf{k})$ имеют полюсы при $\mathbf{k}=i b_{l}$ и $\mathbf{k}=i a_{j}$ соответственно и что благодаря (3.23) и (3.24) вычеты этих функций задаются через их значения в дуальных точках с помощью соотношений

$$
\Phi_{b_{l}}^{\prime}(x)=-i \sum_{j=1}^{N_{a}} \Phi^{\prime}\left(x, i a_{j}\right) c_{j l}, \quad \Psi_{a_{j}}^{\prime}(x)=i \sum_{l=1}^{N_{b}} c_{j l} \Psi^{\prime}\left(x, i b_{l}\right),
$$

как и следовало ожидать. Можно показать, что потенциал $u^{\prime}(x)$ в $(3.22)$ и решения Йоста из (3.23) и (3.24) совпадают с полученными в работе [11], в том числе в рассмотренном здесь случае $N_{a} \neq N_{b}$, который получается при обращении в нуль некоторых строк или столбцов в постоянной матрице $C$, введенной в работе [11]. Мы откладываем рассмотрение условий регулярности потенциала, данного в (3.22), до раздела 5.

\section{4. РЕЗОЛЬВЕНТА}

Коль скоро преобразованный оператор $L^{\prime}$ получен, операторы $\zeta$ и $\eta$ могут быть использованы для анализа его спектральных свойств, а также для исследования вопросов существования и единственности соответствующей резольвенты $M^{\prime}$. Умножая первое и второе уравнения в (3.2) справа на $\eta$ и слева на $\zeta$ соответственно и вспоминая определение (3.5) оператора $P$, получаем сплетающее соотношение

$$
L^{\prime}=\zeta L \eta+L_{\Delta},
$$

где мы ввели

$$
L_{\Delta}=L^{\prime} P=P L^{\prime}
$$

Соответственно, мы ищем резольвенту в виде

$$
M^{\prime}=\zeta M \eta+M_{\Delta},
$$


где произведение $\zeta M \eta$ определено предыдущим построением. Действительно, в силу (2.12) и (2.14) мы получаем, что

$$
\zeta M \eta=\nu^{\prime} M_{0} \omega^{\prime}
$$

а тогда мы имеем для ядра этого произведения в $x$-пространстве следующее билинейное выражение в терминах преобразованных решений Йоста:

$$
\begin{aligned}
(\zeta M \eta)\left(x, x^{\prime} ; q\right)= & -\operatorname{sgn}\left(x_{2}-x_{2}^{\prime}\right) \frac{e^{-q\left(x-x^{\prime}\right)}}{2 \pi} \int d p_{1} \theta\left(\left(q_{2}+p_{1}^{2}-q_{1}^{2}\right)\left(x_{2}-x_{2}^{\prime}\right)\right) \times \\
& \times \Phi^{\prime}\left(x ; p_{1}+i q_{1}\right) \Psi^{\prime}\left(x^{\prime} ; p_{1}+i q_{1}\right)
\end{aligned}
$$

где $\theta(\cdot)$ - функция-ступенька Хевисайда.

Второй член $M_{\Delta}$ в (4.3) подлежит определению. Используя (3.2), (2.9) и (3.5), мы выводим, что $M^{\prime}$ в (4.3) является правым (левым) обратным к $L^{\prime}$ тогда и только тогда, когда $M_{\Delta}$ - решение первого (второго) из операторных уравнений

$$
L^{\prime} M_{\Delta}=P, \quad M_{\Delta} L^{\prime}=P .
$$

Ниже мы рассмотрим разрешимость этих уравнений в случае чисто солитонных потенциалов. Для завершения обсуждения общего случая отметим, что явная форма оператора $P$, определенного в (3.5), может быть получена подстановкой в это равенство операторов $\zeta$ и $\eta$, заданных формулами (3.12) и (3.13). Снова в силу (2.16) мы получаем для хет-ядра этого оператора выражение

$$
\widehat{P}\left(x, x^{\prime} ; q\right)=i \delta\left(x_{2}-x_{2}^{\prime}\right) \sum_{n=1}^{\mathcal{N}} \theta\left(q_{1}-\alpha_{n}\right) \underset{\mathbf{k}=i \alpha_{n}}{\operatorname{res}} \Phi^{\prime}(x, \mathbf{k}) \Psi^{\prime}\left(x^{\prime}, \mathbf{k}\right) .
$$

Здесь мы ввели набор параметров

$$
\left\{\alpha_{1}, \ldots, \alpha_{\mathcal{N}}\right\}=\left\{a_{1}, \ldots, a_{N_{a}}, b_{1}, \ldots, b_{N_{b}}\right\}, \quad \mathcal{N}=N_{a}+N_{b},
$$

чтобы сделать явными свойства симметрии по отношению к использованным ранее параметрам $a_{j}, b_{l}$. Ядро (4.7) отлично от нуля только в интервале значений $q_{1}$ между минимальным и максимальным значениями $\alpha_{m}$. Действительно, в силу (4.7) это очевидно для значений $q_{1}$, лежащих левее указанного интервала, а для значений $q_{1}$, лежащих справа от этого интервала, это следует из равенства

$$
\sum_{n=1}^{\mathcal{N}} \operatorname{res}_{\mathbf{k}=i \alpha_{n}} \Phi^{\prime}(x, \mathbf{k}) \Psi^{\prime}\left(x^{\prime}, \mathbf{k}\right)=0
$$

которое, в свою очередь, является следствием соотношений (3.25). Теперь в соответствии с предыдущим обсуждением мы ожидаем, что $\widehat{M}_{\Delta}$ имеет структуру, подобную $\widehat{P}\left(x, x^{\prime} ; q\right)$. Понятно, что ядро

$$
\widehat{M}_{\Delta}\left(x, x^{\prime} ; q\right)=\mp i \theta\left( \pm\left(x_{2}-x_{2}^{\prime}\right)\right) \sum_{n=1}^{\mathcal{N}} \theta\left(q_{1}-\alpha_{n}\right) \underset{\mathbf{k}=i \alpha_{n}}{\operatorname{res}} \Phi^{\prime}(x, \mathbf{k}) \Psi^{\prime}\left(x^{\prime}, \mathbf{k}\right)
$$


при любом знаке удовлетворяет уравнениям $\mathcal{L}_{x}^{\prime} \widehat{M}_{\Delta}\left(x, x^{\prime} ; q\right)=P\left(x, x^{\prime} ; q\right)$ и $\mathcal{L}_{x^{\prime}}^{\prime d} \widehat{M}_{\Delta}\left(x, x^{\prime} ; q\right)=P\left(x, x^{\prime} ; q\right)$. Но легко видеть, что в общем случае ядро $M_{\Delta}\left(x, x^{\prime} ; q\right)$, построенное с учетом (2.2), растет на пространственной бесконечности, а потому не может быть ядром расширенного оператора в смысле определения из раздела 2. Ниже мы детально рассмотрим эту проблему на специальном примере.

\section{5. ЧИСТО СОЛИТОННЫЙ ПОТЕНЦИАЛ И РЕШЕНИЯ ЙОСТА}

В случае $u(x) \equiv 0$ преобразованный потенциал $u^{\prime}(x)$ есть общий чисто $N$-солитонный потенциал, где $N=\max \left\{N_{a}, N_{b}\right\}$. Этот потенциал и соответствующие решения Йоста можно легко получить из общего представления, найденного в разделе 3. Итак, чисто солитонный потенциал дается любым из равенств в (3.22), где теперь матрица $\mathcal{F}(x)$ имеет элементы

$$
\mathcal{F}_{l j}(x)=\frac{e^{\left(a_{j}-b_{l}\right)\left(x_{1}+\left(a_{j}+b_{l}\right) x_{2}\right)}}{a_{j}-b_{l}} .
$$

Разлагая детерминанты в правой части (3.22) и используя формулу Коши-Бине, получаем, что для регулярности потенциала достаточно, чтобы вещественная матрица $c$ удовлетворяла следующим характеристическим требованиям (эквивалентным тем, что приведены в работе [20]):

$$
\Lambda\left(\begin{array}{c}
l_{1}, l_{2}, \ldots, l_{n} \\
j_{1}, j_{2}, \ldots, j_{n}
\end{array}\right) c\left(\begin{array}{c}
j_{1}, j_{2}, \ldots, j_{n} \\
l_{1}, l_{2}, \ldots, l_{n}
\end{array}\right) \geqslant 0, \quad \Lambda_{l j}=\frac{1}{a_{j}-b_{l}}
$$

для любых $1 \leqslant n \leqslant \min \left\{N_{a}, N_{b}\right\}$ и всех миноров, т.е. для любого выбора $1 \leqslant l_{1}<$ $l_{2}<\cdots<l_{n} \leqslant N_{b}$ и $1 \leqslant j_{1}<j_{2}<\cdots j_{n} \leqslant N_{a}$. Мы использовали здесь стандартное обозначение

$$
A\left(\begin{array}{c}
j_{1}, j_{2}, \ldots, j_{n} \\
l_{1}, l_{2}, \ldots, l_{n}
\end{array}\right)=\operatorname{det}\left\|\begin{array}{cccc}
a_{j_{1} l_{1}} & a_{j_{1} l_{2}} & \ldots & a_{j_{1} l_{n}} \\
a_{j_{2} l_{1}} & a_{j_{2} l_{2}} & \ldots & a_{j_{2} l_{n}} \\
\vdots & \vdots & \ddots & \vdots \\
a_{j_{n} l_{1}} & a_{j_{n} l_{2}} & \ldots & a_{j_{n} l_{n}}
\end{array}\right\|
$$

для миноров матрицы $A=\left\|a_{j l}\right\|$. Формулы (3.23) и (3.24) после достаточно трудоемких вычислений, которые мы здесь опускаем, могут быть более просто представлены как отношения детерминантов. Как и в (3.22), можно использовать альтернативным, но эквивалентным образом детерминанты матриц размера $\left(N_{a} \times N_{a}\right)$ или $\left(N_{b} \times N_{b}\right)$. Мы используем ниже последний выбор, а также симметричное обозначение (4.8). В этих терминах для функций $\chi^{\prime}$ и $\xi^{\prime}$, связанных с решениями Йоста посредством равенств (2.16), мы имеем

$$
\begin{aligned}
& \chi^{\prime}(x, \mathbf{k})=\left(\prod_{l=1}^{N_{b}}\left(b_{l}+i \mathbf{k}\right)^{-1}\right) \frac{\tau_{\chi}(x, \mathbf{k})}{\tau(x)}, \\
& \xi^{\prime}\left(x^{\prime}, \mathbf{k}\right)=\left(\prod_{l=1}^{N_{b}}\left(b_{l}+i \mathbf{k}\right)\right) \frac{\tau_{\xi}\left(x^{\prime}, \mathbf{k}\right)}{\tau\left(x^{\prime}\right)},
\end{aligned}
$$


где $\tau$-функции суть детерминанты:

$$
\begin{aligned}
\tau_{\chi}(x, \mathbf{k}) & =\operatorname{det}\left(\mathfrak{A} e^{\mathcal{A}(x)}(\alpha+i \mathbf{k}) \mathcal{D}\right), \\
\tau_{\xi}\left(x^{\prime}, \mathbf{k}\right) & =\operatorname{det}\left(\mathfrak{A} e^{\mathcal{A}\left(x^{\prime}\right)}(\alpha+i \mathbf{k})^{-1} \mathcal{D}\right), \\
\tau(x) & =\operatorname{det}\left(\mathfrak{A} e^{\mathcal{A}(x)} \mathcal{D}\right)
\end{aligned}
$$

т.е. детерминанты $\left(N_{b} \times N_{b}\right)$-матриц, полученных как произведения квадратных и прямоугольных матриц. А именно,

$$
\begin{aligned}
\mathfrak{A}_{l n} & =\alpha_{n}^{N_{b}-l}, & \alpha+i \mathbf{k} & =\operatorname{diag}\left\{\alpha_{n}+i \mathbf{k}\right\}, \\
e^{\mathcal{A}(x)} & =\operatorname{diag}\left\{e^{\mathcal{A}_{n}(x)}\right\}, & \mathcal{A}_{n}(x) & =\alpha_{n} x_{1}+\alpha_{n}^{2} x_{2}, \\
\mathcal{D} & =\left(\begin{array}{c}
d \\
E_{N_{b}}
\end{array}\right), & d_{j l} & =c_{j l} \frac{\prod_{l^{\prime}=1, l^{\prime} \neq l}^{N_{b}}\left(b_{l}-b_{l^{\prime}}\right)}{\prod_{l^{\prime}=1}^{N_{b}}\left(a_{j}-b_{l^{\prime}}\right)},
\end{aligned}
$$

где $l=1,2, \ldots, N_{b}, j=1,2, \ldots, N_{a}, n=1,2, \ldots, \mathcal{N}$. Если рассматривать потенциал, то он может быть записан как $u^{\prime}(x)=-2 \partial_{x_{1}}^{2} \ln \tau(x)$, и, принимая во внимание последнее соотношение в (5.6), мы восстанавливаем выражение, полученное в работах [19], [20], [22] с использованием тау-функций.

\section{N-СОЛИТОННЫЕ ПОТЕНЦИАЛЫ В СЛУЧАЕ $N_{b}=1$}

Ограничимся специальным случаем $N$-солитонного потенциала с $N_{b}=1$ и произвольным $N_{a}=N$. В дополнение для простоты, но без потери общности мы выбираем $\alpha_{1}<\alpha_{2}<\cdots<\alpha_{\mathcal{N}}$. В этой ситуации потенциал $u^{\prime}(x)$ имеет на $x$-плоскости $N_{a}$ входящих и один исходящий луч, как показано схематично на рисунке. В этом случае $\tau$-функции (5.6) принимают простой вид:

$$
\begin{gathered}
\tau_{\chi}(x, \mathbf{k})=\sum_{m=1}^{\mathcal{N}} f_{m} e^{\mathcal{A}(x)}\left(\alpha_{m}+i \mathbf{k}\right), \quad \tau_{\xi}(x, \mathbf{k})=\sum_{m=1}^{\mathcal{N}} \frac{f_{m} e^{\mathcal{A}(x)}}{\alpha_{m}+i \mathbf{k}}, \\
\tau(x)=\sum_{m=1}^{\mathcal{N}} f_{m} e^{\mathcal{A}(x)}
\end{gathered}
$$

где коэффициенты $f_{m}$ получены из элементов $d_{m 1}$ матрицы $d$ в (5.7) путем перестановки, учитывающей выбранное упорядочение $\alpha_{1}<\alpha_{2}<\cdots<\alpha_{\mathcal{N}}$. Условие $(5.2)$ здесь означает, что все $f_{m}>0$.

Мы хотим показать, что, как сказано во введении, расширенный оператор $L^{\prime}(q)$ с таким потенциалом имеет левый самосопряженный аннигилятор $K(q)$ при значениях $q$, принадлежащих некоторой области на $q$-плоскости. А именно, рассмотрим область внутри вписанного в параболу $q_{2}=q_{1}^{2}$ многоугольника с вершинами в точках с координатами $q_{1}=\alpha_{m}, m=1, \ldots, N$, характеристическая функция которого имеет вид

$$
\begin{aligned}
\kappa(q)= & \sum_{m=1}^{\mathcal{N}-1}\left[\theta\left(q_{1}-\alpha_{m+1}\right)-\theta\left(q_{1}-\alpha_{m}\right)\right] \times \\
& \times\left[\theta\left(q_{2}-\left(\alpha_{m}+\alpha_{m+1}\right) q_{1}+\alpha_{m} \alpha_{m+1}\right)-\theta\left(q_{2}-\left(\alpha_{1}+\alpha_{\mathcal{N}}\right) q_{1}+\alpha_{1} \alpha_{\mathcal{N}}\right)\right] .
\end{aligned}
$$




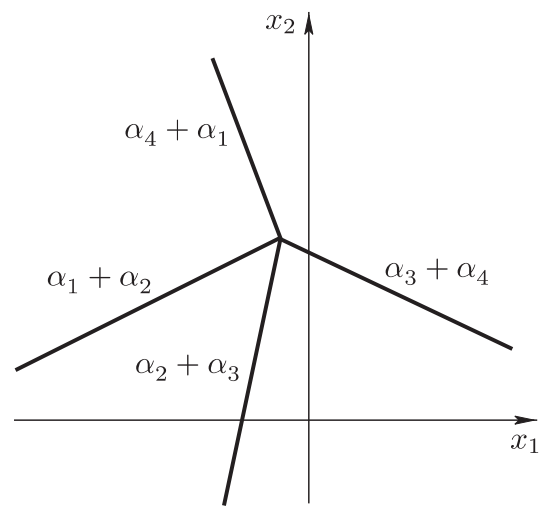

a

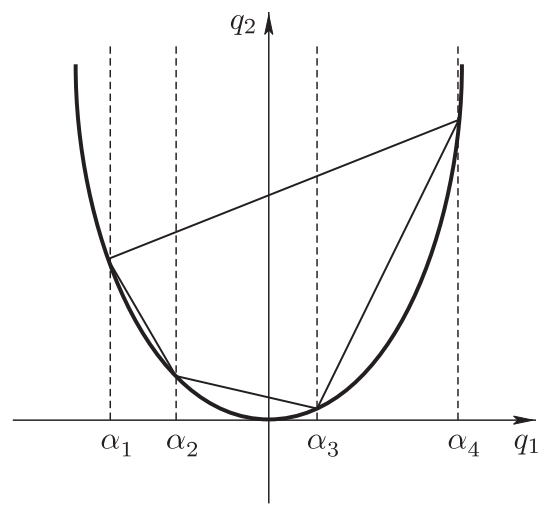

6

Рис. 1. Лучи и многоугольник для $\mathcal{N}=4$.

Заметим, что этот многоугольник можно считать дуальным структуре лучей на $x$-плоскости (см. рисунок для случая $\mathcal{N}=4)$.

Основное наблюдение, необходимое для получения аннигилятора $K(q)$, состоит в том, что функция

$$
\psi(x ; q)=\frac{\kappa(q) e^{q x}}{\tau(x)}
$$

ограничена на $x$-плоскости. Более точно, как можно доказать с помощью более детального рассмотрения, она экспоненциально убывает для $x$, стремящегося к бесконечности по любому направлению на плоскости, при $q$, лежащих внутри многоугольника, а если $q$ принадлежит границе многоугольника, то эта функция имеет направления неубывающего (но ограниченного) поведения.

Вследствие соотношений (2.16), (5.4) и (6.1) функция $1 / \tau$ пропорциональна значению дуального решения Йоста $\Psi^{\prime}(x, \mathbf{k})$ при $\mathbf{k}=i b_{1}$. Тогда мы имеем

$$
\mathcal{L}_{x^{\prime}}^{\prime \mathrm{d}} \hat{\psi}\left(x^{\prime} ; q\right)=0
$$

и, следовательно,

$$
K L^{\prime}=0
$$

для любого оператора $K$ с ядром

$$
K\left(x, x^{\prime} ; q\right)=\varphi(x ; q) \psi\left(x^{\prime} ; q\right)
$$

где $\varphi(x ; q)$ - любая самосопряженная функция, ограниченная по $x$ и тождественно равная нулю вне введенного выше многоугольника.

Можно легко проверить, что $K P=K$. Если мы выбираем $\varphi(x ; q)$ в виде $\varphi(x ; q)=$ $(P \gamma)(x ; q)$, где $\gamma(x ; q)$ - вещественная функция, ограниченная по $x$, равная тождественно нулю вне многоугольника и удовлетворяющая равенству

$$
\int d x \gamma(x ; q) \psi(x, q)=\kappa(q),
$$


то имеем также

$$
P \varphi=\varphi, \quad \int d x \varphi(x ; q) \psi(x, q)=\kappa(q)
$$

Тогда нетрудно показать, что $K$ - самосопряженный проектор, коммутирующий с проектором $P$, т.е. мы имеем

$$
K^{*}=K, \quad K^{2}=K, \quad P K=K P=K .
$$

Существование этого аннигиллятора доказывает, что оператор $L^{\prime}$ не может иметь правый обратный для значений $q$, принадлежащих многоугольнику, определенному характеристической функцией (6.2). Для того чтобы доказать, что, напротив, резольвента $M^{\prime}(q)$ существует для $q$, лежащих вне многоугольника, обратимся к хет-ядру $\widehat{M}_{\Delta}\left(x, x^{\prime} ; q\right)$, определенному в $(4.10)$, и рассмотрим свойства ограниченности $M_{\Delta}\left(x, x^{\prime} ; q\right)=e^{-q\left(x-x^{\prime}\right)} \widehat{M}_{\Delta}\left(x, x^{\prime} ; q\right)$. Используя $(2.16),(5.4),(5.5)$ и $(6.1)$, мы записываем явно эту функцию как

$$
\begin{aligned}
M_{\Delta}\left(x, x^{\prime} ; q\right)= & \frac{ \pm e^{-q\left(x-x^{\prime}\right)} \theta\left( \pm\left(x_{2}-x_{2}^{\prime}\right)\right)}{\tau(x) \tau\left(x^{\prime}\right)} \times \\
& \times \sum_{m, n=1}^{\mathcal{N}} f_{m} f_{n} \theta\left(q_{1}-\alpha_{m}\right)\left(\alpha_{m}-\alpha_{n}\right) e^{\mathcal{A}_{m}(x)+\mathcal{A}_{n}(x)} .
\end{aligned}
$$

В силу (4.9) эта функция тождественно равна нулю вне полосы $\alpha_{1}<q_{1}<\alpha_{N+1}$ на $q$-плоскости. Внутренняя же часть полосы делится многоугольником на части, лежащие сверху и снизу от многоугольника. Тогда, выбирая верхний (нижний) знак для $q$, лежащих над (под) многоугольником в этой полосе, можно доказать, что ядро $M_{\Delta}\left(x, x^{\prime} ; q\right)$ - ограниченная функция от $x, q$ и что она определяет ядро расширенного оператора $M_{\Delta}(q)$ в соответствии с определением из раздела 2. Мы заключаем, что для $q$, не принадлежащих многоугольнику, $M_{\Delta}(q)$ удовлетворяет уравнениям (4.6), и, следовательно, резольвента $M^{\prime}(q)$ существует в этой области и дается формулой (4.3).

Заметим, что функция Грина $G\left(x, x^{\prime}, \mathbf{k}\right)$ оператора $\mathcal{L}^{\prime}$ может быть определена (см. работы [11], [12]) как значение ядра $M^{\prime}\left(x, x^{\prime} ; q\right)$ при $q_{1}=\mathbf{k}_{\Im}, q_{2}=\mathbf{k}_{\Im}^{2}-\mathbf{k}_{\Re}^{2}$ для комплексного спектрального параметра $\mathbf{k}=\mathbf{k}_{\Re}+i \mathbf{k}_{\Im}$. Эти значения $q$ лежат вне параболы $q_{2}=q_{1}^{2}$, а потому вне многоугольника и касаются его только в вершинах, так что функция Грина существует для любого значения $\mathbf{k}$, но сингулярна в точках $\mathbf{k}=i \alpha_{m}$, отвечающих вершинам многоугольника. Это единственные сингулярности функции Грина, поскольку разрывы первого члена в (4.3) при $q_{1}=\alpha_{m}$ (см. (4.5)) скомпенсированы (вне многоугольника) разрывами второго члена, как следует из равенства (4.10).

Наконец, уместно отметить, что случай $N_{a}=1$ и произвольного $N_{b}$ может быть рассмотрен аналогично, при этом вместо левого для $L^{\prime}(q)$ получается правый аннигилятор для $q$ внутри многоугольника, и в случае $N_{a}=N_{b}=1$ многоугольник сводится к сегменту на линии $q_{2}=\left(\alpha_{1}+\alpha_{2}\right) q_{1}-\alpha_{1} \alpha_{2}$ с конечными точками $q_{1}=\alpha_{1}$ и $q_{1}=\alpha_{2}$. В этом случае восстанавливаются результаты, полученные в работе [11]. 
Благодарности. Эта работа частично поддержана РФФИ (грант № 08-01-00501, совместные гранты РФФИ-КЭ № 06-01-92057 и NWO-РФФИ № 047.011.2004.059), Программой поддержки ведущих научных школ (грант НШ-795.2008.1), Программой РАН "Математические методы нелинейной динамики", а также INFN и Консорциумом E.I.N.S.T.E.I.N. A.K. Погребков благодарит факультет физики Университета Саленто (Лечче) за теплое гостеприимство.

\section{Список литературы}

[1] В. С. Дрюма, Писъма в ЖЭТФ, 19:12 (1974), 753-755.

[2] В. Е. Захаров, А.Б. Шабат, Функи. анализ и его прил., 8:3 (1974), 43-53.

[3] M. J. Ablowitz, D. Bar Yaacov, A. S. Fokas, Stud. Appl. Math., 69:2 (1983), 135-143.

[4] П. Г. Гриневич, С. П. Новиков, Функи. анализ и его прил., 22:1 (1988), 23-33.

[5] M. Boiti, F. Pempinelli, A. K. Pogrebkov, M. C. Polivanov, Inverse Problems, 8:3 (1992), $331-364$.

[6] М. Бойти, Ф. Пемпинелли, А.К. Погребков, М.К. Поливанов, ТМФ, 93:2 (1992), $181-210$.

[7] M. Boiti, F. Pempinelli, A. K. Pogrebkov, J. Math. Phys., 35:9 (1994), 4683-4718.

[8] M. Boiti, F. Pempinelli, A. K. Pogrebkov, Inverse Problems, 13:3 (1997), L7-L10.

[9] М. Бойти, Ф. Пемпинелли, А. К. Погребков, Б. Принари, ТМФ, 116:1 (1998), 3-53.

[10] B. Prinari, Inverse Problems, 16:3 (2000), 589-603.

[11] M. Boiti, F. Pempinelli, A. Pogrebkov, B. Prinari, Inverse Problems, 17:4 (2001), 937-957.

[12] M. Boiti, F. Pempinelli, A. K. Pogrebkov, B. Prinari, J. Math. Phys., 43:2 (2002), 1044-1062.

[13] М. Бойти, Ф. Пемпинелли, А. К. Погребков, Б. Принари, ТМФ, 144:2 (2005), 257-276.

[14] М. Бойти, Ф. Пемпинелли, А. К. Погребков, Б. Принари, “Спектральная теория нестационарного уравнения Шрёдингера с двумерно возмущенным одномерным потенциалом", Нелинейная динамика, Тр. МИАН, 251, ред. Е. Ф. Мищенко, 2005, 10-53.

[15] J. Villarroel, M. J. Ablowitz, Stud. Appl. Math., 109:3 (2002), 151-162.

[16] M. Boiti, F. Pempinelli, A. K. Pogrebkov, J. Phys. A, 39:8 (2006), 1877-1898.

[17] M. Boiti, F. Pempinelli, A. K. Pogrebkov, J. Math. Phys., 47:12 (2006), 123510.

[18] S. V. Manakov, V. E. Zakharov, L. A. Bordag, A. R. Its, V. B. Matveev, Phys. Lett. A, 63:3 (1977), 205-206.

[19] G. Biondini, Y. Kodama, J. Phys. A, 36:42 (2003), 10519-10536.

[20] G. Biondini, S. Chakravarty, J. Math. Phys., 47:3 (2006), 033514.

[21] G. Biondini, Phys. Rev. Lett., 99:6 (2007), 064103.

[22] S. Chakravarty, Y. Kodama, J. Phys. A, 41:27 (2008), 275209. 